\section{Aplicação da mortalidade hospitalar após a realização de cirurgia de revascularização do miocárdio para monitoramento do cuidado hospitalar}

\author{
Use of hospital mortality rates following \\ coronary artery bypass graft surgery \\ to monitor hospital care
}

\author{
José Carvalho de Noronha 1,2 \\ Mônica Martins 3 \\ Claudia Travassos 1 \\ Mônica R. Campos 3 \\ Paula Maia 1 \\ Rogério Panezzuti
}

\footnotetext{
1 Centro de Informação Científica e Tecnológica, Fundação Oswaldo Cruz, Rio de Janeiro, Brasil.

2 Instituto de Medicina Social, Universidade do Estado do Rio de Janeiro, Rio de Janeiro, Brasil.

3 Escola Nacional de Saúde Pública, Fundação Oswaldo Cruz, Rio de Janeiro, Brasil.

Correspondência J. C. Noronha

Departamento de Informação em Saúde, Centro de Informação Científica e Tecnológica, Fundação Oswaldo Cruz. Av. Brasil 4365, Rio de Janeiro, $R J$ 21045-900, Brasil. noronha@cict.fiocruz.br
}

\begin{abstract}
Brazilian hospitals performing coronary artery by-pass graft surgery (CABG) from 1996 to 1998 and covered by the Ministry of Health were ranked according to their risk-adjusted hospital mortality rates. Seventy-six hospitals that performed more than 150 CABGs (total of 38,962 surgical interventions) were classified as low or high outliers according to the ratio between observed and expected hospital mortality rates. Overall hospital mortality rate was $7.20 \%$. The rate was $3.48 \%$ among patients treated in the low outliers and $13.96 \%$ among the high outliers. The methodology was useful for discriminating Brazilian hospitals according to their post-CABG mortality rates and may be a useful tool for identifying hospitals with possible quality-of-care problems.
\end{abstract}

Health Services; Myocardial Revascularization; Hospital Mortality; Surgery
A taxa de mortalidade hospitalar tem sido utilizada, como um indicador de resultados, para a avaliação da qualidade dos cuidados prestados, para alguns procedimentos médico-cirúrgicos nos quais a morte não é um evento raro (Nightingale, 1863, apud Fink et al. 1; Codman, 1916, apud Normand et al. 2) 3,4,5,6,7. A cirurgia de revascularização do miocárdio, utilizada amplamente para o tratamento da doença coronariana desde os trabalhos pioneiros de Favaloro ${ }^{8}$, constitui-se em um desses casos.

A utilização e a divulgação de dados de mortalidade hospitalar após cirurgia de revascularização do miocárdio, para comparação de desempenho de hospitais e cirurgiões, foram empregadas de maneira pioneira pelo Departamento de Saúde do Estado de Nova Iorque, Estados Unidos, com o desenvolvimento de um registro de casos, o Cardiac Surgery Reporting System. Os primeiros dados foram divulgados em dezembro de 1990 9, e a primeira divulgação pública ocorreu em dezembro de 199210. Pouco tempo depois, essa iniciativa foi estendida aos Estados da Pensilvânia e Nova Jérsei, e seguida por outros esforços colaborativos, como o de Cleveland e o do Consórcio da Nova Inglaterra Setentrional 11.

Em 1999, sistema similar migrou da literatura médica para o público em geral no Canadá 12. No Reino Unido, a Sociedade de Cirurgiões Cardiotorácicos da Grã-Bretanha e Irlanda iniciou um projeto piloto em 1994 e publi- 
cou seu primeiro relatório sobre 12 hospitais em 1996. O relatório de 2000-2001 já incluía $86,00 \%$ dos hospitais do Serviço Nacional de Saúde do país (http://www.scts.org/index.cfm? ukcardiacreg=yes, acessado em 26/Jan/2004).

Um dos problemas centrais na utilização de dados de resultados, como a mortalidade hospitalar, para comparação do desempenho entre prestadores, que enseja grande debate, é a necessidade de se promover um ajuste de linha de base que dê conta das diferenças de gravidade dos pacientes atendidos. Caso contrário, prestadores com altas taxas de mortalidade poderiam protestar alegando que seus pacientes eram "mais doentes" 13. De fato, as diferenças nas taxas de mortalidade entre hospitais ou médicos podem ser atribuídas a três grupos de fatores: diferenças quanto à gravidade do estado de saúde da população atendida em cada hospital; qualidade dos cuidados pode ser inferior em alguns hospitais por problemas estruturais ou nos processos de cuidado (adequação do cuidado prestado e da tecnologia empregada); e erros aleatórios 14. Para a cirurgia de revascularização do miocárdio, existe uma vasta literatura explorando diversos modelos para se proceder a esse ajuste de risco. Jones et al. 15 relatam o resultado de um consenso, realizado com a participação dos principais grupos dos Estados Unidos integrantes do Projeto Cooperativo de Base de Dados para cirurgia de revascularização do miocárdio (Cooperative Coronary Artery By-pass Surgery Database Project), para identificação e hierarquização de um conjunto de variáveis clínicas úteis para o monitoramento e redução da mortalidade precoce de pacientes submetidos à cirurgia de revascularização do miocárdio. Vários estudos têm se seguido analisando diferentes modelos de ajuste de risco para previsão de óbitos e complicações após a cirurgia de revascularização do miocárdio 13,16,17,18,19,20,21,22,23.

Tu et al. 16 compararam o impacto de modelos mais abrangentes em relação àqueles que contêm um número limitado de fatores de risco considerados cruciais. Para isso, avaliaram o impacto incremental da introdução dos fatores de risco nos modelos, utilizando as variáveis nucleares e de nível um do painel descrito por Jones et al. 15. O seu trabalho evidenciou que, após a inclusão de seis variáveis, o ganho no poder discriminatório do modelo, avaliado pela área sob a curva ROC, é muito pequeno. As variáveis que agregaram maior poder discriminatório foram idade (65-74, $\geq 75$ ), sexo feminino, cirurgia de emergência, cirurgia coronária prévia, insuficiência ventricular esquerda grau 3 e 4 (frações de ejeção, respectivamente, de 20,00 a $34,00 \%$ e $<20,00 \%$ ), e doença de tronco da coronária esquerda (estenose $\geq 50,00 \%$ ).

Neste artigo, apresenta-se um estudo voltado para classificar os hospitais vinculados ao Sistema Único de Saúde (SUS) do Brasil com base no seu desempenho para a realização de cirurgia de revascularização do miocárdio. A medida de desempenho empregada teve como base a taxa de mortalidade hospitalar, ajustada pelo risco de morrer, calculada a partir das variáveis disponíveis no Sistema de Informações Hospitalares do SUS (SIH-SUS).

\section{Metodologia}

\section{População estudada}

Os dados foram obtidos dos hospitais brasileiros que realizaram cirurgia de revascularização do miocárdio pagas pelo Ministério da Saúde, através do SIH-SUS, nos anos de 1996 a 1998. Na criação da base de dados da pesquisa, utilizaram-se os arquivos dos CD-ROMs mensais de 1996, 1997 e 1998 24, selecionando-se apenas os registros cujo procedimento realizado tinha o código "32011016" (cirurgia coronariana com circulação extracorpórea). Os casos de cirurgia de revascularização do miocárdio realizada sem a utilização de circulação extracorpórea também foram codificados como tal pela ausência de codificação específica à época do estudo. Da mesma forma, não foi possível identificar a realização de outros procedimentos cardíacos associados. Os hospitais foram identificados pelo seu número de inscrição no Cadastro Geral de Contribuintes. Como os dados das Autorizações de Internação Hospitalar (AIH), após processamento, estão disponíveis em diferentes arquivos com estruturas diversas, foi necessário o desenvolvimento de um processo de integração de dados descrito por Campos et al. 25. As variáveis disponíveis estão descritas no Manual da AIH 26. A variável dependente utilizada foi "motivo da cobrança", recodificada em óbito e não óbito.

Noronha 21 discute em detalhes os critérios de seleção das variáveis do estudo. A variável “caráter da internação", que caracterizaria a emergência ou não da cirurgia, apesar da relevância como fator de risco, não pôde ser utilizada por forte inconsistência na qualificação 
das situações de emergência. A maior parte das internações $(67,10 \%)$ foi qualificada como de emergência, ainda que realizadas por hospitais que não dispunham desse tipo de atendimento. Não foi possível, com os dados disponíveis, estabelecer qualquer validação dessa variável. Travassos \& Martins 27 já haviam demonstrado a baixa confiabilidade de tal informação.

O emprego da variável "diagnóstico principal” como indicativo de gravidade também se mostrou inviável, uma vez que os registros apresentavam alto grau de imprecisão. Foi observado que vários hospitais "padronizavam" seus diagnósticos principais, isto é, o mesmo diagnóstico era atribuído a todos os procedimentos de "cirurgia coronária com circulação extracorpórea”. Também houve escasso lançamento de outros diagnósticos - diagnósticos secundários - $(55,80 \%)$, ainda assim de maneira muito assistemática em relação aos diferentes hospitais.

Não foi possível, também, a identificação dos cirurgiões que realizaram o procedimento pelos seus registros de Cadastro de Pessoas Físicas (CPF) por três razões básicas. Primeiro, porque não era autorizado o lançamento de CPFs em hospitais públicos e universitários. Em segundo lugar, porque, para uma proporção significativa de hospitais, havia o lançamento de códigos de faturamento diretamente para o hospital, o que não permitia a identificação do profissional. E, finalmente, porque havia regularidade no lançamento de um mesmo CPF para várias AIHs, aparentemente mais relacionado à burocracia de emissão da AIH do que ao cirurgião ou ao crédito de honorários a um cirurgião específico, independentemente do fato de ter ou não participado da cirurgia.

Através do emprego de regressão logística e testes de poder discriminatório 28 , foi ajustado um modelo de predição de óbito com as variáveis: idade (categorias menos de 40, 40-59 e 60 e mais anos) e sexo. Esse modelo não apresentou problemas de ajuste (teste de $\chi^{2}$ Hosmer Lemeshow $=1,928 ; \mathrm{p}=0,587$ ), e capacidade de discriminação medida pela curva ROC foi 0,586 (IC95\%: 0,576-0,597) 21.

Com base nesse modelo, foram calculadas as probabilidades de morte para cada paciente, e as probabilidades preditas, somadas para se chegar ao número esperado de mortes em cada hospital. Calculou-se, então, a razão entre o número observado (O) e o esperado (E) de óbitos para cada hospital (razão O/E), que foram, em seguida, ordenados em ordem crescente de acordo com os valores das razões obtidas. Os hospitais foram classificados como desviantes altos ou baixos, correspondendo aos percentis 20 e 80 . Esses cálculos foram efetua- dos apenas para os hospitais que realizaram mais de 150 cirurgias durante os três anos estudados.

\section{Resultados}

De janeiro de 1996 a dezembro de 1998, foram pagas pelo Ministério da Saúde 41.989 internações, realizadas em 131 hospitais localizados em 22 Unidades da Federação, codificadas com o procedimento "cirurgia coronária com circulação extracorpórea”. A maioria dos pacientes operados no período foi do sexo masculino $(67,50 \%)$. A idade média foi de 59,9 anos, com desvio padrão de 10,1 anos. O tempo de permanência foi de 14,5 dias, em média, com desvio padrão de 12,3 dias. A média do tempo de permanência em Unidade de Terapia Intensiva (UTI) foi de 3,8 dias, com desvio padrão de 4,0 dias. A taxa global de mortalidade hospitalar registrada no período foi de $7,20 \%$.

Os pacientes que faleceram eram mais velhos (62,9 contra 59,7 anos), apresentaram um tempo médio de permanência no hospital inferior (11,5 contra 14,7 dias), permaneceram mais dias na UTI (5,5 contra 3,7 dias) e apresentaram maior valor médio de internação (R\$ $5.114,00$ comparados com R \$ 4.702,00). Doze por cento dos óbitos ocorreram nas primeiras 48 horas de internação. A maior parte dos óbitos ocorreu nos primeiros dias de internação, apresentando uma distribuição adernada para a esquerda, com tempos de permanência mais curtos, quando comparada com a distribuição das altas. Quanto à permanência em UTI, evidencia-se uma distribuição mais alargada dos óbitos, com um pique nos primeiros dias, prolongados nos tempos de permanência maior quando superam as altas. Estas se concentram nos tempos entre três a nove dias de permanência em UTI.

Dos 131 hospitais que realizaram cirurgia coronária, o ajuste de risco por idade e sexo foi efetuado para $76(58,00 \%)$ que realizaram mais de 150 cirurgias no período estudado. Esses hospitais realizaram 38.962 cirurgias coronárias, correspondendo a $92,10 \%$ do total de cirurgias estudadas. Os hospitais identificados como desviantes baixos (razão $\mathrm{O} / \mathrm{E}<1$; percentil 20) realizaram 7.607 cirurgias $(19,50 \%$ do total). Os desviantes altos (razão O/E > 1; percentil 80) realizaram 9,60\% do tal de cirurgias (3.731) (Tabela 1).

A taxa de mortalidade hospitalar para o grupo de pacientes operados nos hospitais desviantes baixos foi de $3,48 \%$. Para os pacientes dos hospitais desviantes altos, a taxa foi de 
Taxas de mortalidade hospitalar após cirurgia de revascularização do miocárdio experimentadas pelos conjuntos de pacientes operados nos hospitais desviantes baixos (razão O/E < 1) e altos (razão O/E > 1). SIH-SUS, Brasil, 1996-1998.

\begin{tabular}{lcccccc}
\hline Desviantes & $\begin{array}{c}\text { Números de } \\
\text { internações }\end{array}$ & $\begin{array}{c}\text { Volume } \\
\text { médio }\end{array}$ & $\begin{array}{c}\text { Variação do } \\
\text { volume }\end{array}$ & $\begin{array}{c}\text { Número } \\
\text { de óbitos }\end{array}$ & $\begin{array}{c}\text { Taxas } \\
\text { Variação } \\
\text { das taxas }\end{array}$ \\
\hline Baixos (percentil 20) & 7.607 & 507 & $159-1.698$ & 265 & $3,48 \%$ & $1,64-4,53$ \\
Altos (percentil 80) & 3.731 & 248 & $151-419$ & 521 & $13,96 \%$ & $11,52-17,28$
\end{tabular}

Razão O/E = razão entre o número observado e o número esperado de óbitos para cada hospital; SIH-SUS = Serviço de Informações Hospitalares do Sistema Único de Saúde.

13,96\% (Tabela 1). A razão de chance de morrer nos hospitais desviantes altos em relação aos baixos foi de 4,011. Caso os hospitais desviantes altos experimentassem as taxas de mortalidade média para o Brasil, teriam sido evitados 260 óbitos, e, caso experimentassem as taxas dos desviantes baixos, a cifra seria de 390 óbitos evitados.

Os hospitais que realizaram mais de 150 cirurgias nos três anos de estudos, ordenados em ordem crescente de acordo com as razões entre o número observado de óbitos hospitalares e o número esperado calculados pela soma das probabilidades de óbito (razão O/E), são apresentados nas Tabelas 2, 3 e 4 .

\section{Discussão}

Nosso estudo evidenciou acentuada diferença nas taxas de mortalidade hospitalar entre os dois grupos de desviantes. A metodologia empregada foi capaz de discriminar os hospitais brasileiros com relação à mortalidade pós cirurgia de revascularização do miocárdio e constitui-se em um instrumento útil para identificação dos hospitais vinculados aos SUS com possíveis problemas de qualidade, apesar de suas limitações decorrentes das restrições dos dados disponíveis no SIH-SUS para ajuste de risco.

Thomas \& Hofer 5 efetuaram extensa revisão sobre a validade das taxas de mortalidade ajustadas por risco como medida da qualidade da assistência hospitalar. Concluíram que há indícios suficientes para demonstrar que os hospitais que prestam assistência de baixa qualidade apresentam maiores taxas de mortalidade ajustadas por riscos do que os outros hospitais. Entretanto, afirmam que não podem os pacientes estar confiantes de que hospitais com taxas de mortalidade significativamente mais baixas do que as esperadas sejam, de fato, pres-
Tabela 2

Relação dos hospitais que realizaram cirurgia de revascularização do miocárdio desviantes baixos (percentil 20), ordenados pelas razões O/E. SIH-SUS, Brasil, 1996-1998.

\begin{tabular}{|c|c|c|c|c|c|}
\hline Hospital & $\begin{array}{l}\text { Número } \\
\text { de casos }\end{array}$ & $\begin{array}{l}\text { Número } \\
\text { de óbitos }\end{array}$ & $\begin{array}{c}\text { Número } \\
\text { de óbitos } \\
\text { esperados }\end{array}$ & $\begin{array}{c}\text { Taxa de } \\
\text { mortalidade } \\
\text { bruta }\end{array}$ & Razão O/E \\
\hline 14 & 183 & 3 & 13 & 1,64 & 0,23 \\
\hline 32 & 497 & 10 & 34 & 2,01 & 0,29 \\
\hline 18 & 406 & 11 & 29 & 2,71 & 0,38 \\
\hline 49 & 627 & 17 & 44 & 2,71 & 0,39 \\
\hline 73 & 538 & 15 & 38 & 2,79 & 0,39 \\
\hline 19 & 289 & 9 & 22 & 3,11 & 0,41 \\
\hline 61 & 387 & 12 & 28 & 3,10 & 0,42 \\
\hline 64 & 399 & 12 & 27 & 3,01 & 0,44 \\
\hline 9 & 284 & 10 & 20 & 3,52 & 0,51 \\
\hline 76 & 1.083 & 38 & 74 & 3,51 & 0,51 \\
\hline 75 & 488 & 19 & 34 & 3,89 & 0,56 \\
\hline 12 & 212 & 9 & 15 & 4,25 & 0,59 \\
\hline 67 & 357 & 16 & 26 & 4,48 & 0,62 \\
\hline 41 & 1.698 & 77 & 123 & 4,53 & 0,63 \\
\hline 20 & 159 & 7 & 11 & 4,40 & 0,64 \\
\hline
\end{tabular}

Razão O/E = razão entre o número observado e o número esperado de óbitos para cada hospital; SIH-SUS = Serviço de Informações Hospitalares do Sistema Único de Saúde.

tadores de boa qualidade. Igualmente, demonstram, com base na literatura revisada, que não há como afirmar que as diferenças nas taxas de mortalidade ajustadas para dois hospitais quaisquer indiquem diferenças na qualidade do desempenho.

A nossa pesquisa demonstrou a utilidade de indicadores simples de resultados como a mortalidade hospitalar para comparar o desempenho de hospitais para o caso da cirurgia de revascularização do miocárdio. No caso da cirurgia de revascularização do miocárdio, é 
Tabela 3

Relação dos hospitais que realizaram cirurgia de revascularização do miocárdio desviantes altos (percentil 80), ordenados pelas razões O/E. SIH-SUS, Brasil, 1996-1998.

\begin{tabular}{lccccc}
\hline Hospital & $\begin{array}{c}\text { Número } \\
\text { de casos }\end{array}$ & $\begin{array}{c}\text { Número } \\
\text { de óbitos }\end{array}$ & $\begin{array}{c}\text { Número } \\
\text { de óbitos } \\
\text { esperados }\end{array}$ & $\begin{array}{c}\text { Taxa de } \\
\text { mortalidade } \\
\text { bruta }\end{array}$ & Razão O/E \\
\hline 23 & 187 & 22 & 13 & 11,76 & 1,72 \\
62 & 356 & 41 & 24 & 11,52 & 1,72 \\
4 & 306 & 38 & 21 & 12,42 & 1,79 \\
30 & 227 & 28 & 15 & 12,33 & 1,81 \\
26 & 166 & 20 & 11 & 12,05 & 1,83 \\
53 & 205 & 27 & 15 & 13,17 & 1,85 \\
25 & 333 & 44 & 24 & 13,21 & 1,87 \\
40 & 419 & 55 & 29 & 13,13 & 1,89 \\
58 & 173 & 25 & 13 & 14,45 & 1,95 \\
74 & 152 & 21 & 11 & 13,82 & 1,98 \\
8 & 187 & 26 & 13 & 13,90 & 1,99 \\
24 & 314 & 48 & 22 & 15,29 & 2,19 \\
16 & 151 & 24 & 11 & 15,89 & 2,23 \\
35 & 303 & 48 & 20 & 15,84 & 2,36 \\
56 & 167 & 29 & 12 & 17,37 & 2,45 \\
2 & 272 & 47 & 19 & 17,28 & 2,46 \\
\hline
\end{tabular}

Razão O/E = razão entre o número observado e o número esperado de óbitos para cada hospital; SIH-SUS = Serviço de Informações Hospitalares do Sistema Único de Saúde. identificarmos fragilidades no modelo que empregamos para o ajuste de risco. Entretanto, é possível que a magnitude dos problemas de qualidade em nossos hospitais e grande variabilidade no desempenho na cirurgia de revascularização do miocárdio entre hospitais expliquem o fato de o modelo de ajuste de risco utilizado, apesar de suas limitações, ter sido capaz de discriminar os serviços.

Peterson et al. 20 procuraram avaliar a precisão preditiva de quatro modelos de risco clínico de mortalidade após cirurgia de revascularização do miocárdio e examinar em que medida os resultados cirúrgicos dos hospitais, ajustados por risco, dependiam do método de ajuste de risco utilizado. Aplicaram seus modelos a 3.654 pacientes submetidos à cirurgia de revascularização do miocárdio em 28 hospitais do Alabama e Iowa, nos Estados Unidos. Embora os quatro modelos de risco tenham revelado capacidade discriminatória semelhantes, eles tenderam a superestimar a mortalidade dos pacientes com maior risco de morrer. O aspecto importante deste trabalho foi o de aplicar modelos desenvolvidos em outras bases de dados a uma base diferente daquela para as quais haviam sido desenvolvidos. Os dados sugerem que a aplicação de diferentes modelos de ajuste de risco resulta em medidas de desempenho relativas semelhantes ao nível de cada hospital, isto é, se o hospital apresentava um bom desempenho quando comparado com seus pares, usando um dos modelos de ajuste risco, ele também tinha uma grande probabilidade de apresentar um desempenho superior qualquer que fosse o modelo de ajuste de risco utilizado. Ainda segundo os autores, estas conclusões são consistentes com os achados de outros estudos semelhantes e devem diminuir as aflições dos clínicos de que seus desempenhos diferenciados possam ser de alguma forma um artefato do método de ajuste de risco aplicado. Mais adiante, afirmam ter encontrado uma correlação muito mais forte entre dois métodos quaisquer de ajuste de risco de resultados do que entre qualquer método de ajuste risco e dados não ajustados, indicando que alguma forma de ajuste de risco é necessária para comparação apropriada. Além disso, reconhecem os autores, se o que se deseja é comparar hospitais, o mesmo modelo de ajuste de risco deve ser aplicado a todos os hospitais estudados.

O outro aspecto a ser ressaltado é o elevado grau de proximidade crescentemente demonstrado entre a precisão preditiva dos modelos estatísticos e dos modelos dos clínicos de resultados após cirurgia de revascularização do miocárdio, recentemente demonstrado por Iva- 
nov et al. 19. Esses autores compararam as estimativas de probabilidade de mortalidade hospitalar e tempo de permanência por mais de 48 horas após cirurgia coronária em unidades de cuidados intensivos, com estimativas derivadas de modelos estatísticos. A previsão dos clínicos foi desenvolvida com e sem acesso à regra preditiva. Não houve diferença entre as previsões com e sem regra. O conjunto das previsões dos clínicos foi comparado com as previsões dos modelos estatísticos e também não foi possível ser observada grande diferença entre as duas estimativas. $\mathrm{Na}$ realidade, os clínicos sobreestimaram a probabilidade de mortalidade por mais de 48 horas. Suas previsões não foram significativamente maiores do que a do modelo para os não sobreviventes, mas foi significativamente maior para os sobreviventes.

Essas considerações fazem-nos recomendar que sejam feitos aprimoramentos na qualidade dos bancos de dados administrativos com a incorporação de um pequeno número de variáveis, mas com grande contribuição para o ajuste do risco de morrer após a cirurgia de revascularização do miocárdio 16. A simples inclusão de informações sobre a fração de ejeção, a história de cirurgia anterior e a existência de doença de tronco agregariam informações relevantes para um melhor ajuste de risco para os hospitais.

Porém, considera-se que a identificação de hospitais desviantes altos e baixos com a metodologia que empregamos representa uma abordagem que possibilita orientar, no momento, esforços dirigidos à melhoria do desempenho dos centros de cirurgia coronariana. Para os hospitais que se encontram na faixa de desviantes altos, um esforço deve ser empreendido para identificar possíveis problemas de qualidade. Aqueles que se encontram nas faixas inferiores não devem se descuidar de seus esforços de melhoria, podendo, entretanto, servir de marcos de referência para todos os centros que buscam um aprimoramento da qualidade dos serviços que prestam, da segurança e dos pacientes que atendem.

Recomenda-se também que os hospitais sejam encorajados a preencher adequadamente os campos já atualmente existentes nas AIHs, como a natureza da internação e diagnósticos principal e secundários. Uma definição mais clara dessas variáveis melhoraria a confiabilidade dos dados. Os clínicos e cirurgiões, administradores e outros profissionais de saúde serão beneficiados pelo exame regular dessas informações.

Deve-se buscar formas de identificação do médico responsável pela cirurgia no SIH-SUS,
Tabela 4

Relação dos hospitais que realizaram cirurgia de revascularização do miocárdio não desviantes (entre percentis 20 e 80), ordenados pelas razões O/E. SIH-SUS, Brasil, 1996-1998.

\begin{tabular}{|c|c|c|c|c|c|}
\hline Hospital & $\begin{array}{l}\text { Número } \\
\text { de casos }\end{array}$ & $\begin{array}{l}\text { Número } \\
\text { de óbitos }\end{array}$ & $\begin{array}{l}\text { Número } \\
\text { de óbitos } \\
\text { esperados }\end{array}$ & $\begin{array}{c}\text { Taxa de } \\
\text { mortalidade } \\
\text { bruta }\end{array}$ & Razão O/E \\
\hline 7 & 659 & 30 & 46 & 4,55 & 0,66 \\
\hline 55 & 6.407 & 314 & 450 & 4,90 & 0,70 \\
\hline 11 & 325 & 16 & 23 & 4,92 & 0,71 \\
\hline 50 & 453 & 23 & 32 & 5,08 & 0,71 \\
\hline 52 & 400 & 20 & 27 & 5,00 & 0,73 \\
\hline 27 & 174 & 9 & 12 & 5,17 & 0,75 \\
\hline 51 & 241 & 13 & 17 & 5,39 & 0,75 \\
\hline 22 & 245 & 13 & 17 & 5,31 & 0,76 \\
\hline 66 & 788 & 45 & 58 & 5,71 & 0,77 \\
\hline 72 & 228 & 12 & 15 & 5,26 & 0,78 \\
\hline 42 & 437 & 24 & 30 & 5,49 & 0,79 \\
\hline 31 & 658 & 38 & 47 & 5,78 & 0,80 \\
\hline 47 & 199 & 12 & 14 & 6,03 & 0,87 \\
\hline 59 & 194 & 12 & 14 & 6,19 & 0,87 \\
\hline 37 & 924 & 59 & 65 & 6,39 & 0,91 \\
\hline 43 & 1.875 & 123 & 133 & 6,56 & 0,92 \\
\hline 17 & 169 & 11 & 12 & 6,51 & 0,93 \\
\hline 69 & 504 & 34 & 35 & 6,75 & 0,97 \\
\hline 15 & 1.055 & 76 & 77 & 7,20 & 0,99 \\
\hline 46 & 213 & 15 & 15 & 7,04 & 0,99 \\
\hline 63 & 485 & 36 & 34 & 7,42 & 1,05 \\
\hline 71 & 473 & 36 & 34 & 7,61 & 1,07 \\
\hline 39 & 322 & 25 & 23 & 7,76 & 1,10 \\
\hline 28 & 1.054 & 83 & 75 & 7,87 & 1,11 \\
\hline 68 & 291 & 23 & 21 & 7,90 & 1,12 \\
\hline 36 & 532 & 43 & 37 & 8,08 & 1,15 \\
\hline 38 & 193 & 15 & 13 & 7,77 & 1,16 \\
\hline 70 & 393 & 33 & 28 & 8,40 & 1,17 \\
\hline 1 & 223 & 17 & 14 & 7,62 & 1,19 \\
\hline 54 & 166 & 14 & 12 & 8,43 & 1,21 \\
\hline 48 & 243 & 21 & 17 & 8,64 & 1,23 \\
\hline 60 & 510 & 46 & 36 & 9,02 & 1,27 \\
\hline 33 & 1.347 & 126 & 97 & 9,35 & 1,31 \\
\hline 29 & 854 & 80 & 59 & 9,37 & 1,35 \\
\hline 10 & 182 & 18 & 13 & 9,89 & 1,36 \\
\hline 45 & 375 & 36 & 26 & 9,60 & 1,38 \\
\hline 13 & 378 & 39 & 28 & 10,32 & 1,41 \\
\hline 44 & 542 & 55 & 38 & 10,15 & 1,44 \\
\hline 3 & 442 & 43 & 30 & 9,73 & 1,45 \\
\hline 6 & 232 & 23 & 16 & 9,91 & 1,46 \\
\hline 57 & 169 & 19 & 12 & 11,24 & 1,58 \\
\hline 5 & 460 & 53 & 32 & 11,52 & 1,63 \\
\hline 34 & 225 & 26 & 16 & 11,56 & 1,66 \\
\hline 65 & 503 & 59 & 35 & 11,73 & 1,67 \\
\hline 21 & 415 & 50 & 29 & 12,05 & 1,71 \\
\hline
\end{tabular}

Razão $\mathrm{O} / \mathrm{E}$ = razão entre o número observado e o número esperado de óbitos para cada hospital; SIH-SUS = Serviço de Informações Hospitalares do Sistema Único de Saúde. 
ainda que, para efeitos de cobrança, o crédito seja efetuado ao hospital.

Grande esforço deve ser desenvolvido para a melhoria dos prontuários clínicos. Em 1919, o Colégio Americano de Cirurgiões já estabelecia para seu minimum standard que prontuários precisos e completos deviam ser preenchidos para todos os pacientes 30 . Nos bancos das escolas de medicina, os estudantes são treinados a preenchê-los de maneira adequada. Mas são seus próprios mestres que ensinam, na sua prática clínica quotidiana, como esquecer o que aprenderam nos bancos escolares. É um grande equívoco atribuir a esse requisito básico o apodo de "burocracia". Prontuários adequados e completos constituem-se na melhor ferramenta para que se possa ter um acompanhamento e um controle adequados da prática profissional. A padronização dos documentos de internação, o estabelecimento de rotinas para seu preenchimento e a implantação de formulários de resumos de alta apropriados são de grande relevância para a melhoria da qualidade dos nossos serviços de saúde.

Hannan et al. 31 estão convencidos de que a divulgação pública de dados sobre desempenho dos hospitais tem se constituído em um forte estímulo para iniciativas de melhoria de qualidade e contribuiu significativamente para a queda da mortalidade observada, sobretudo nos primeiros anos de divulgação. Os resultados continuam a ser processados e divulgados 10. A expansão da iniciativa para o Reino Unido (http://www.scts.org/index.cfm?ukcardiacreg=yes, acessado em 26/Jan/2004) demonstra que o sistema veio para ficar. Os usuários dos serviços de saúde tornam-se cada vez mais exigentes, e financiadores públicos e privados buscam avaliar a qualidade do emprego de seus recursos. Chassin 32 acredita que a divulgação pública é necessária na maior parte das vezes. Examinando a experiência acumulada pelo Estado de Nova Iorque, cujas taxas de mortalidade hospitalar caíram $41,00 \%$ durante os primeiros quatro anos do programa e continuam caindo, ele defende a divulgação. Não apenas pelo argumento de que as pessoas têm direito à informação, mas, sobretudo, porque a divulgação dos dados transformou-se em uma força poderosa para a melhoria dos cuidados: mobilizou os médicos, oferecendo-lhes, pela primeira vez, dados comparativos de desempenho e impulsionou os hospitais na busca de maiores e melhores recursos para melhorar seus serviços de cirurgia cardíaca.

Hannan et al. 11 estudaram os efeitos da divulgação de resultados de mortalidade hospitalar ajustada por fatores de risco pré-operató- rios após cirurgia de revascularização do miocárdio em pacientes do Medicare entre 1994 e 1999. Quatro das cinco regiões estadunidenses com programas de qualidade com disseminação pública de dados apresentaram taxas de mortalidade hospitalar significativamente menores do que para o resto do país, para cada ano e para os seis anos em conjunto. Os autores tiveram o cuidado de testar a hipótese de que as regiões estudadas pudessem estar evitando pacientes de alto risco, não apenas pelo ajuste de risco, mas também comparando a prevalência total e as tendências de prevalência desses pacientes. Não puderam encontrar nenhuma evidência de que isso estivesse acontecendo. Concluem os autores que iniciativas formais de melhoria de qualidade, associadas à disseminação pública de dados de resultados, parecem estar ligadas a menores taxas de mortalidade hospitalar após cirurgia de revascularização do miocárdio. Em nosso estudo, por ser a primeira iniciativa do gênero no país, decidimos omitir os nomes dos hospitais que apresentavam taxas discrepantes, a maior e a menor. Entretanto, não demorará a hora em que esses dados, preferencialmente melhor colhidos, ajustados e trabalhados, deverão vir a público.

\section{Conclusões}

Há muito que ser aproveitado das bases de dados do SIH-SUS para estudos dos serviços hospitalares. Apesar das limitações existentes, elas permitem discriminar hospitais, para aquelas condições em que a mortalidade hospitalar não é um evento raro, como o caso da cirurgia de revascularização do miocárdio, com o uso apenas de variáveis demográficas. Torna-se evidente, no entanto, que esses bancos devem ser aprimorados para permitir o melhor emprego de seus dados na avaliação de desempenho dos serviços. Por outro lado, este estudo apenas indica as diferenças na mortalidade entre hospitais, e estudos específicos que analisem as causas das diferenças de mortalidade hospitalar após cirurgia de revascularização do miocárdio devem ser fortemente encorajados em nosso país. 


\section{Resumo}

Este estudo classificou os hospitais vinculados ao Sistema Único de Saúde (SUS) do Brasil com base no desempenho para a realização de cirurgia de revascularização do miocárdio, entre 1996 e 1998, com o uso da taxa de mortalidade hospitalar ajustada pelo risco de morrer. Foram estudados 76 hospitais $(58,00 \%$ do total) que realizaram mais de 150 cirurgias no período, correspondentes a 38.962 cirurgias $(92,10 \%$ do total), que foram classificados como desviantes altos ou baixos, de acordo com a razão entre o número observado e o esperado de óbitos para cada hospital. A taxa global de mortalidade hospitalar foi de 7,20\%. Para o grupo de pacientes operados nos hospitais desviantes baixos, foi de 3,48\%, e, de 13,96\% para os desviantes altos. A metodologia tem utilidade para discriminar os hospitais brasileiros com relação à mortalidade pós cirurgia de revascularização do miocárdio e pode ser um instrumento útil para identificação daqueles que possam apresentar problemas de qualidade.

Serviços de Saúde; Revascularização Miocárdia; Mortalidade Hospitalar; Cirurgia

\section{Colaboradores}

J.C. Noronha foi responsável pela concepção da pesquisa. Em conjunto com M. Martins e C. Travassos participaram do desenvolvimento da pesquisa, revisão bibliográfica, análise dos dados e redação. C. Travassos colaborou na revisão final do artigo. O. M. R. Campos participou da montagem dos bancos e análise estatísticas dos dados. P. Maia e R. Panezzutti participaram da revisão bibliográfica e discussão dos resultados.

\section{Referências}

1. Fink A, Yano EM, Brook RH. The condition of the literature on differences in hospital mortality. Med Care 1989; 27:315-36

2. Normand ST, Glickman ME, Sharma RG, McNeil BJ. Using admission characteristics to predict short-term mortality from myocardial infarction in elderly patients. Results from the Cooperative Cardiovascular Project. JAMA 1996; 275:1322-8.

3. Dubois RW, Brook RH, Rogers WH. Adjusted hospital death rates: a potential screen for quality of medical care. Am J Public Health 1987; 77:1162-7.

4. Office of Technology Assessment. The quality of medical care: information for consumers, OTAH-386. Washington DC: United States Government Printing Office; 1988.

5. Thomas JW, Hofer TP. Research evidence on the validity of risk-adjusted mortality rate as a measure of hospital quality of care. Med Care Res Rev 1998; 55:371-404.

6. Travassos CM, Noronha JC, Martins MS. Mortalidade hospitalar como indicador de qualidade: uma revisão. Ciênc Saúde Coletiva 1999; 4:36781.

7. Martins MS. Durée de séjour et mortalité hospitalière au Brésil: comparaison entre hôpitaux publics et privés [PhD Thesis]. Montreal: Faculté de Médecine, Université de Montreal; 2003.

8. Favaloro RG. Surgical treatment of coronary arteriosclerosis. Baltimore: Williams \& Wilkins; 1970.

9. Hannan EL, Kilburn Jr. H, O’Donnell JF, Lukacik G, Shields EP. Adult open heart surgery in New York State. JAMA 1990; 264:2768-74.

10. New York State Department of Health. Coronary artery bypass surgery in New York State: 1989-1991. Albany: New York State Department of Health; 1992.

11. Hannan EL, Sarrazin MSV, Doran DR, Rosenthal GE. Provider profiling and quality improvement efforts in coronary artery by-pass graft surgery: the effect on short-term mortality among Medicare beneficiaries. Med Care 2003; 41:1164-72.

12. Ghali WA, Rothwell DM, Quan H, Brant R, Tu JV. A Canadian comparison of data sources for coronary artery bypass surgery outcome "report cards". Am Heart J 2000; 140:402-8.

13. Landon B, Iezzoni LI, Ash AA, Shwartz M, Daley J, Hughes JS, et al. Judging hospitals by severity-adjusted mortality rates: the case of CABG surgery. Inquiry 1996; 33:155-66.

14. Iezzoni L. Risk adjustment for measuring health care outcomes. Ann Arbor: Health Administration Press; 1994.

15. Jones RH, Hannan EL, Hammermeister KE, Delong ER, O'Connor GT, Luepker RV, et al. Identification of preoperative variables needed for risk adjustment of short-term mortality after coronary artery bypass graft surgery. The Working Group Panel on the Cooperative CABG Database Project. J Am Coll Cardiol 1996; 28:1478-87.

16. Tu JV, Sykora K, Naylor CD. Assessing the outcomes of coronary artery bypass graft surgery: how many risk factors are enough? J Am Coll Cardiol 1997; 30:1317-23.

17. Bridgewater B, Neve H, Moat N, Hooper T, Jones 
M. Predicting operative risk for coronary artery surgery in the United Kingdom: a comparison of various risk prediction algorithms. Heart 1998; 79:350-5.

18. Iezzoni LI, Ash AS, Shwartz M, Landon BE, MacKiernan Y. Predicting in-hospital deaths from coronary artery bypass graft surgery. Do different severity measures give different predictions? Med Care 1998; 36:3-5.

19. Ivanov J, Borger MA, David TE, Cohen G, Walton N, Naylor CD. Predictive accuracy study: comparing a statistical model to clinicians' estimates of outcomes after coronary bypass surgery. Ann Thorac Surg 2000; 70:162-8.

20. Peterson ED, Delong ER, Muhlbaier LH, Rosem AB, Buell HE, Kiefe CI, et al. Challenges in comparing risk-adjusted bypass surgery results: results from the Cooperative Cardiovascular Project. J Am Coll Cardiol 2000; 36:2174-84.

21. Noronha JC. Utilização de indicadores de resultados para a avaliação da qualidade em hospitais de agudos: mortalidade hospitalar após cirurgia de revascularização do miocárdio em hospitais brasileiros [Tese de Doutorado]. Rio de Janeiro: Instituto de Medicina Social, Universidade do Estado do Rio de Janeiro; 2001.

22. Bridgewater B, Grayson AD, Jackson M, Brooks N, Grotte GJ, Keenan DJM, et al. Surgeon specific mortality in adult cardiac surgery: comparison between crude and risk stratified data. BMJ 2003; 327:13-7.

23. Shroyer AL, Coombs LP, Peterson ED, Eiken MC, Delong ER, Chen A, et al. The Society of Thoracic Surgeons: 30-day operative mortality and morbidity risk models. Ann Thorac Surg 2003; 75:185665.
24. Departamento de Informação e Informática do SUS. Movimento de autorização de internação hospitalar: arquivos mensais. Períodos: janeiro 1996 a dezembro de 1998 [CD-ROM]. Brasília: Ministério da Saúde; 1999.

25. Campos MR, Martins M, Noronha JC, Travassos C. Proposta de integração de dados do Sistema de Informações Hospitalares do Sistema Único de Saúde (SIH-SUS) para pesquisa. Inf Epidemiol SUS 2000; 9:51-8.

26. Ministério da Saúde. Manual da AIH. Rio de Janeiro: Departamento de Informação e Informática do SUS; 2000.

27. Travassos C, Martins M. A confiabilidade dos dados nos formulários de Autorização de Internação Hospitalar (AIH), Rio de Janeiro, Brasil. Cad Saúde Pública 1994; 10:339-55.

28. Hosmer D, Lemeshow S. Applied logistic regression. New York: John Wiley \& Sons; 1989.

29. Thomas JW, Hofer TP. Accuracy of risk-adjusted mortality rate as a measure of hospital quality of care. Med Care 1999; 37:83-92.

30. Brauer CM. Champions of quality in health care: a history of the joint commission on accreditation of healthcare organizations. Lyme: Greenwich Publishing Group; 2001.

31. Hannan EL, Kilburn Jr. H, Racz M, Shields E, Chassin MR. Improving the outcomes of coronary artery bypass surgery in New York State. JAMA 1994; 271:761-6.

32. New York State Department of Health. Coronary artery bypass surgery in New York State: 19971999. Albany: New York State Department of Health; 2002.

33. Chassin MR. Achieving and sustaining improved quality: lessons from New York State and cardiac surgery. Health Aff 2002; 21:40-51.

Recebido em 06/Abr/2004

Versão final reapresentada em 03/Set/2004 Aprovado em 09/Set/2004 